\title{
PREVALENCIA DEL CONSUMO DE CIGARRO Y OTROS PRODUCTOS DE TABACO ENTRE EL PERSONAL DOCENTE QUE LABORA EN LAS INSTITUCIONES EDUCATIVAS DE NIVEL SECUNDARIO - DISTRITO DE TACNA- 2005"
}

Responsable: Lic. Elide Tipacti Sotomayor Miembro: QF. Noelia Flores Tipacti

\author{
RESUMEN
}

Se presenta un estudio descriptivo transversal que tiene como objetivo principal determinar la prevalencia del consumo de cigarros y otros productos de tabaco entre el personal docente que labora en instituciones educativas de nivel secundario del distrito de Tacna -2005. Se plantea que al ser considerados modelos de los estudiantes podrian indirectamente fomentar el inicio del consumo de cigarro en los mismos. Se pretende dar a conocer cuál es la situación y obtener información preliminar para futuras investigaciones.

En una muestra de 314 docentes, se aplicó un cuestionario destinado a la obtención de respuestas sobre el problema y características del investigado a partir del propio sujeto en cuestión. Los resultados muestran que el $51,37 \%$ de docentes son ex fumadores y de los docentes que fuman, el $61,9 \%$ corresponde a la categoría de fumadores leves; el $6,8 \%$ a la categoría de fumadores moderados. Solo el $6,23 \%$ de docentes reconoce que ha fumado estando en la escuela. Se concluye que la prevalencia del consumo de cigarro y otros productos de tabaco es del $37,6 \%$.

Palabras clave: Consumo de cigarro; tabaco; prevalencia; colegio de nivel secundario; docente.

\section{ABSTRACT}

It is presented a cross-sectional descriptive study, which main goal is determining the prevalence of cigarettes and other kind of tobacco consumption among the staff who work in teaching institutions in a high school level in Tacna during 2005. It is exposed that as they are considered models for the students, they could indirectly foment the beginning of cigarettes consumption in students. It is pretended to reveal which is the situation and to gather preliminary information for future investigations.

In a sample of 314 teachers, a questionnaire was applied, designed to get answers about the problem and the characteristics of the investigated subject starting from himself / herself. The results show that $51.37 \%$ of the teachers are ex-smokers. The $61.9 \%$ of the teachers who smoke belong to the light smokers category, the $6.8 \%$ belong to the moderate smokers category, and only $6.23 \%$ of the teachers accept they had smoked while they were at school. The conclusion is that prevalence of cigarettes and other kind of tobacco consumption is 37.6 percent.

Key words: cigarettes consumption; tobacco; prevalence; High school level; teacher.

\section{INTRODUCCIÓN}

El consumo de tabaco es conocido desde hace más de dos mil años, pero en el siglo $X X$ alcanza el carácter de pandémico, constituyéndose en un grave problema sanitario a nivel mundial. Es en la última década, donde se ha podido tener una noción más clara de la severidad de la epidemia. En el año 2000 se estimó en 4,9 millones de muertes por año, lo cual constituyó un crecimiento del $45 \%$ con respecto a lo calculado para 1990. El incremento fue mayor para los paises no desarrollados, esperándose que se duplique para el año 2020. En el año 2002, casi 40 de cada 100 peruanos fumaron tabaco.

En muchos países, sobre todo del mundo en desarrollo, se está comenzando a fumar a edades cada vez más tempranas, con frecuencia antes de cumplir los 15 años. Por lo anterior, la prevalencia de fumadores es frecuentemente alta entre adolescentes.
En el Perú, también se observa una tendencia a iniciar el consumo de drogas legales e ilegales a edades cada vez más tempranas. Las generaciones más jóvenes se están iniciando más temprano en el consumo de las diferentes drogas.

La mitad de los adolescentes varones ya ha consumido tabaco a los 15 años, mientras que la mitad de las mujeres ha iniciado consumo a los 16 . A los 19 años, 9 de cada 10 adolescentes ya han consumido tabaco. El grupo de mayor riesgo para el inicio de consumo de tabaco se ubica entre los 12 y 17 años.

En los jóvenes de 13 a 15 años el uso actual de cigarrillos es alto $(23,4 \%$ en Lima vs. $17,4 \%$ en provincias); el uso de otros productos derivados del tabaco es bajo $(8,8 \%$ en Lima vs. $6,6 \%$ en el resto urbano).

El problema del tabaquismo, por tanto, compromete de lleno a toda la comunidad 
educacional. Las escuelas son un lugar ideal para hacer una labor preventiva. La educación es, en sí misma, preventiva: ella forma valores, actitudes, habilidades y conductas que permiten al niño, niña y joven desarrollarse en forma integral, anticiparse a la aparición de problemas y aprender a enfrentar los riesgos del medio social donde se desenvuelve. En este contexto, el profesor ocupa un lugar primordial debido al desarrollo de sus funciones, entre las que se encuentra, en la actualidad, el abordaje de la prevención de las drogas, motivando al alumnado a no experimentar con el tabaco y a mantenerse sin empezar a fumar: y ayudando a los que ya fuman a que dejen de hacerlo. Sin embargo, y dado que uno de cada tres adultos fuma, es probable que exista un alto consumo de tabaco entre los docentes de nivel primario y secundario, $y$ al ser éstos considerados modelos de los estudiantes podrian indirectamente fomentar el inicio del consumo de tabaco en los adolescentes.

Dentro de este marco de referencia, se ha creído por conveniente estudiar la prevalencia del consumo de cigarro y otros productos de tabaco entre los docentes, dada la urgente necesidad de realizar actividades preventivas para evitar que los jóvenes comiencen a fumar a edades cada vez más tempranas, ya que una vez que el sujeto se ha iniciado, el poder altamente adictivo de la nicotina se encarga de que el hábito se mantenga y que con el tiempo el sujeto se vea privado de la libertad suficiente para dejar de fumar.

El primer objetivo de la prevención es que los adolescentes nunca lleguen a consumir tabaco, aquellos que lo han probado no lo vuelvan a hacer, aquellos que lo consumen ocasionalmente en lo posible que no lo consuman y que quienes tengan consumo abusivo reciban el apoyo adecuado para que lo dejen, al igual que quienes son dependientes.

\section{OBJETIVOS DE LAINVESTIGACIÓN}

1. Determinar la prevalencia del consumo de cigarros y otros productos de tabaco entre el personal docente que labora en las instituciones educativas de nivel secundario del distrito de Tacna.

2.Identificar las características sociodemográficas, por tipo de colegio, del personal docente que fuma o fumó cigarro y otros productos de tabaco.

3. Establecer la clasificación en materia de tabaquismo por tipo de colegio del personal docente que fuma o fumó cigarro y otros productos de tabaco.

\section{MATERIAL Y MÉTODOS}

La población estuvo constituida por 1711 docentes de instituciones educativas de nivel secundario, tanto públicas (1315) como privadas
(396) del distrito de Tacna en el año 2005.

Para definir la muestra, las instituciones educativas se seleccionaron mediante muestreo estratificado simple proporcional. Para cada estrato se procedió a la elección de la muestra usando el azar simple. Las unidades de observación se seleccionaron mediante muestreo no probabilístico (grupos intactos asignados por aulas seleccionadas al azar).

Tamaño total de la muestra: 314 docentes, 189 de colegios estatales y 125 de colegios particulares, con los siguientes criterios de inclusión:

- Docentes de las instituciones educativas secundarias seleccionadas que se encuentren laborando en el año académico 2005 al inicio del estudio.

- Que residan en el distrito de Tacna.

- Aquellos que de manera voluntaria acepten llenar el instrumento.

El instrumento que se empleó fue la encuesta. Se aplicó a través de un cuestionario estructurado y autoadministrado.

\section{RESULTADOS}

Los hallazgos nos muestran que el $81,8 \%$ del personal docente ha fumado alguna vez, correspondiendo el $62,64 \%$ a los docentes de colegios estatales y el $37,36 \%$ a los de colegios particulares. El grupo que predomina es el de 30 a 39 años con énfasis en los de sexo femenino, del cual el $48,14 \%$ son de colegios estatales y el $19,44 \%$ son de colegios particulares (ver Cuadro 1). El tabaquismo es un fenómeno social de primera magnitud, la elevada capacidad aditiva de la nicotina hace que pueda considerarse como una auténtica drogadicción y también como una enfermedad crónica.

CUADRO 1.Personal Docente que Labora en Instituciones Educativas de Nivel Secundario que ha Fumado alguna vez, por Edad Según Tipo de Colegio y Sexo

\begin{tabular}{|c|c|c|c|c|c|c|c|c|c|c|}
\hline \multirow{4}{*}{ EDAD } & \multicolumn{8}{|c|}{ TIPO DE COLEGIO } & \multirow{3}{*}{\multicolumn{2}{|c|}{ TOTAL }} \\
\hline & \multicolumn{4}{|c|}{ ESTATAL } & \multicolumn{4}{|c|}{ PARTICULAR } & & \\
\hline & \multicolumn{2}{|c|}{ MASCULINO } & \multicolumn{2}{|c|}{ FEMENINO } & \multicolumn{2}{|c|}{ MASCULINO } & \multicolumn{2}{|c|}{ FEMENINO } & & \\
\hline & $N^{0}$ & $\%$ & $N^{0}$ & $\%$ & $\mathrm{~N}$ & $\%$ & $N$ & $\%$ & \multirow[t]{2}{*}{$\mathrm{N}$} & \multirow[t]{2}{*}{$\%$} \\
\hline$<24$ & & & & & & & & & & \\
\hline $25-29$ & 6 & 16,22 & 11 & 29,73 & 7 & 18,92 & 13 & 35,13 & 37 & 100 \\
\hline $30-39$ & 26 & 24,1 & 52 & 48,14 & 9 & 8,33 & 21 & 19,44 & 108 & 100 \\
\hline $40-49$ & 11 & 18,03 & 36 & 59,02 & 5 & 8,2 & 9 & 14,75 & 61 & 100 \\
\hline 50.59 & 9 & 23,68 & B & 21,05 & 9 & 23,68 & 12 & 31,6 & 38 & 100 \\
\hline $60+$ & 2 & 15,35 & & & 5 & 38,5 & 6 & 46,15 & 13 & 100 \\
\hline Total & 54 & 21,01 & 107 & 41,63 & 35 & 13,62 & 61 & 23,74 & 257 & 100 \\
\hline
\end{tabular}


GRÁFICO $N^{\circ}$ 1. Personal Docente que Labora en Instituciones Educativas de Nivel Secundario que Actualmente Fuma Diariamente, Ocasionalmente o no Fuma

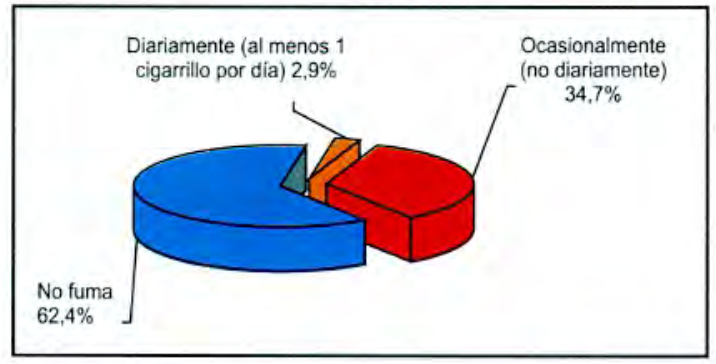

Actualmente tenemos, según el Gráfico $N^{\circ} 1$ que el $2,9 \%$ de personal docente fuma diariamente al menos un cigarrillo por día, pero también observamos que el $34,7 \%$ fuma ocasionalmente, es decir, no diariamente, tanto los de colegios estatales como de colegios particulares. De acuerdo con estos datos podemos decir que existe una prevalencia del $37,6 \%$ de docentes que consumen cigarro y otros productos de tabaco. Una idea errónea, frecuente, es considerar al tabaquismo como un simple hábito, como el morderse las uñas; es, en realidad, una dependencia, pues la nicotina, principio activo del tabaco, es una droga adictiva y, como tal, tiene las características de otras drogas como dependencia física y psicológica. Esta dependencia está reconocida como un trastorno mental y de comportamiento por la OMS.

El consumo de tabaco es la principal causa prevenible de mortalidad a nivel mundial, y ocasiona cinco millones de muertes por año. Según datos probatorios, fumar es nocivo para casi todos los órganos del cuerpo. Es la causa del $90 \%$ de los casos de cáncer de pulmón y está asociado a muchos otros tipos de cáncer, como el cervical o el renal, así como con enfisema, bronquitis, asma y otras enfermedades respiratorias. Entre otros riesgos sanitarios asociados al consumo de tabaco figuran tipos de cáncer como los de boca, garganta y cuello, así como ataques cardiacos, accidentes cerebrovasculares, otras enfermedades cardiovasculares e infertilidad.

Siendo las escuelas un lugar ideal para hacer labor preventiva, ya que en ellas se forman valores, es necesario anticiparse a la aparición de problemas, y en este contexto el profesor ocupa un lugar primordial ya que una de sus funciones en la actualidad es el abordaje de la prevención de drogas, motivando a los alumnos a mantenerse sin empezar a fumar.

GRÁFICO $N^{\circ}$ 2. Personal Docente que Labora en Instituciones Educativas de Nivel Secundario según Unidades en Promedio que Fuma por dia

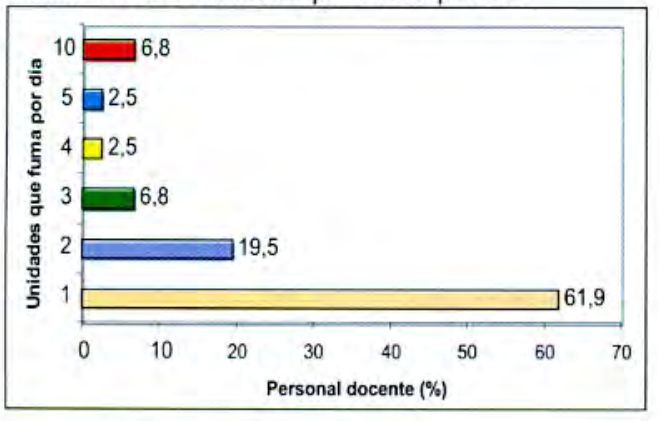

Si bien no existe unanimidad acerca de a quiénes debemos considerar fumadores leves, moderados o severos, ni del límite exacto que separa al fumador habitual del esporádico, en los trabajos científicos suelen aparecer rangos de clasificación, pero estos son variables, en todo caso podría llamarse fumador leve a aquellos que fuman menos de 10 cigarrillos y moderado a aquellos entre 10 y 20 . Entonces, de acuerdo con la clasificación en materia de tabaquismo según los hallazgos encontrados, tenemos que del $45,91 \%$ que corresponde al personal docente que fuma actualmente, al $6,8 \%$ se le categoriza como fumadores moderados porque fuman en promedio más de 10 unidades por dia, siendo el $62,5 \%$ de éstos de colegios particulares; asi mismo, se les categoriza como fumadores leves al $61,9 \%$ de docentes que fuman actualmente porque consumen menos de 10 unidades diarias, tanto los de colegios estatales como los de particulares como lo muestra el Gráfico $\mathrm{N}^{\circ} 2$.

Considerando como fumador pasivo a la persona que convive con el fumador moderado o leve, todos los alumnos de los colegios estatales y particulares que tienen profesores fumadores recaen en esta categoría. Estos estudiantes son los que tienen mayor probabilidad de enfermarse que los no fumadores, puesto que la persona vecina al fumador que aspira durante 1 hora el humo es como si hubiera fumado 2 a 3 cigarrillos. Los fumadores que respiran en un ambiente de fumadores se convierten en fumadores pasivos.

Al consumir un cigarrillo se producen dos tipos de corrientes de humo: la primera (corriente principal) es aquella que, al aspirar una calada, pasa por el interior del cigarrillo hasta alcanzar los pulmones del fumador activo; la segunda (corriente secundaria) es la que se desprende al ambiente desde el extremo incandescente del cigarrillo que surge de la combustión directa del tabaco y que puede ser inhalada por un sujeto pasivo que respira en ese entorno contaminado. La nocividad de esta corriente secundaria para el fumador pasivo actualmente está fuera de toda duda.

GRÁFICO $\mathbf{N}^{\circ}$ 3. Personal Docente que Labora en Instituciones Educativas de Nivel Secundario Según Tiempo que dejó de Fumar

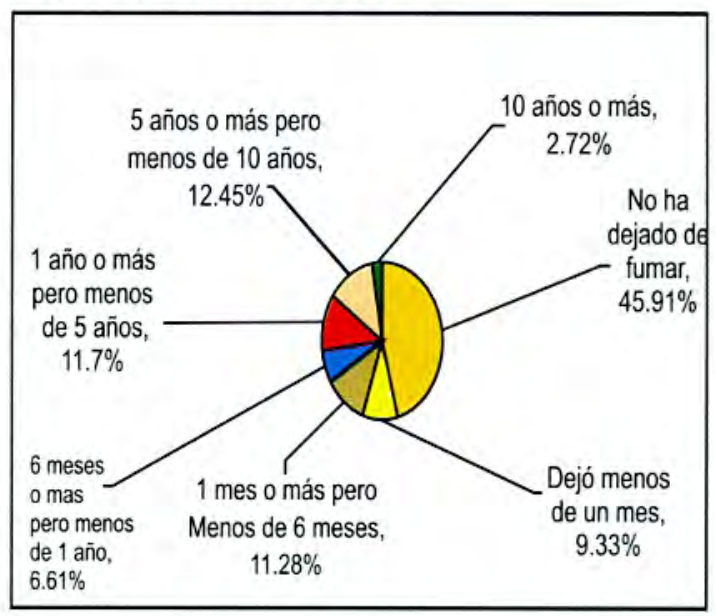


En la categoria de ex fumadores, que son aquellas personas que han dejado de fumar un mes o más al momento de la encuesta, según el Gráfico $N^{\circ} 3$ tenemos que el $51,37 \%$ de docentes que fumaban, hace menos de 10 años han dejado de fumar, y el $2,72 \%$ hace más de 10 años ha dejado de hacerlo.

\section{v. CONCLUSIONES}

1. La prevalencia del consumo de cigarros y otros productos de tabaco entre el personal docente que labora en instituciones educativas de nivel secundario del distrito de Tacna es de $37,6 \%$.

2. Del total de docentes de nivel secundario que fuman o han fumado alguna vez $(81,8 \%)$, el $62,64 \%$ son de colegios estatales, de los cuales el $21,01 \%$ corresponden al sexo masculino y el $41,63 \%$ al sexo femenino. El $37,36 \%$ del total de docentes que fuman o han fumado son de colegios particulares, de los cuales el $13,62 \%$ corresponden al sexo masculino y el $23,74 \%$ al sexo femenino.

3. Respecto a la edad tenemos que el grupo etareo comprendido entre 30 y 39 años tiene el $42 \%$ de personal docente que fuma o ha fumado alguna vez, en los cuales predomina el sexo femenino con el $48,14 \%$ de colegios estatales y el $19,44 \%$ de colegios particulares.

4. Referente a la clasificación en materia de tabaquismo del personal docente que fuma concluimos que el $45,91 \%$ son actualmente fumadores y un $54,09 \%$ son ex fumadores.

5. El $18,2 \%$ del total de docentes de nivel secundario son no fumadores.

6. De los docentes que fuman, $6,8 \%$ son fumadores moderados con mayor porcentaje en docentes de colegios particulares y $93,2 \%$ son fumadores leves sobre todo de colegios estatales.

7. Los estudiantes a cargo de los docentes que representan el $37,6 \%$ de fumadores, son considerados fumadores pasivos y tienen la mayor probabilidad de enfermarse sufriendo los efectos del tabaco.

8. El $6,23 \%$ de los docentes fumadores acepta que ha fumado alguna vez en la escuela, convirtiéndose asi en modelos de sus alumnos para el consumo de cigarros a temprana edad.

\section{BIBLIOGRAFÍA}

Champagne, Beatriz. "El Problema del tabaco y su solución: el Convenio Marco para el Control del Tabaco" en línea. En: Fundación Interamericana del Corazón. Tercer Congreso Virtual de Cardiología. $<$ www.fac.org.ar/tcvc/public/tabaco.html> Consulta: 11 feb. 2005.

Centro Nacional de Información y Comunicación Educativa. La Prevención del tabaquismo en linea. Madrid.

<http://www.cnice.mecd.es/recursos/primaria/transv ersales/tabaquismo.htm> Consulta: 11 feb. 2005.

Colegio de Profesores de Chile. Informe estudio de salud laboral de los profesores en Chile.en línea.<http://www.colegiodeprofesores.cl/nacional/ informe\%20salud.pdf >Consulta: 9 feb. 2005.

Consumer en línea.

<http://www.consumer.es/web/es/noticias/salud_y seguridad/2004/12/07/113047. php> Consulta: 15 feb. 2005.

Convenio Marco de la OMS para el Control del

Tabaco en línea.

$<H$ ttp://www.who.int/tobacco/fctc/text/en/fctc_es.p df> Consulta: 9 feb. 2005.

"Día mundial sin tabaco" en línea. El Mundo $<$ http://elmundosalud.elmundo.es/elmundosalud/e speciales/diasin/muertes.htm> Consulta: 15 feb. 2005

Encuesta Nacional de Prevención y Consumo de Drogas 2002 DEVIDA en línea.<http://www. pnufid.org.pe/publicaciones/ii\%20encuesta $\% 20 \%$ 202002.pdf> Consulta: 9 feb. 2005.

Organización Mundial de la Salud. El cuerpo del fumador en línea. Ginebra. 2001.

$<$ http://www.who.int/tobacco/en/smokers_es_po.p df>Consulta: 11 feb. 2005.

Organización Panamericana de la Salud. Mitos y verdades sobre el tabaco. en linea. Julio 2005 <http://www.paho.org/Spanish/AD/SDE/RA/toh_gr eatest hits.pdf> Consulta: 11 julio. 2005.

Prieto, Ernesto . "Programa de cese de tabaco" en línea. En: Asociación Argentina de Medicina Respiratoria. Buenos Aires. 2004.

<http://www.aamr.org.ar/index.php?option=com_c ontent\&task=view\&id=95\&ltemid=261>Consulta: abril. 2005.

Saludalia. en linea. Madrid

$<$ http://www.saludalia.com/docs/Salud/web_salud alia/temas_de_salud/doc/tabaquismo/doc/doc_def inicion_concepto2.htm> Consulta: 11 marzo. 2005.

Sharma R. "Tobacco use among school personnel in Rajasthan, India." en linea. Indian Journal of Cancer. Vol 41, Nro. 4, 2004. pp. $162-$ 166.

<http://www.nhicindia.org/site/Search/HicsJournal s.asp?Topic $=$ Adult $\% 20$ Health \&Type $=$ Health $\% 20 \mathrm{~T}$ opic\&ICat=Scientific\%20Articles> Consulta: mayo 2005.

Sinha, DN. "Tobacco use among school personnel in eight North-eastern states of India" en línea. Indian Journal of Cancer. Vol. 40, No. 1 , 2003 , pp. 3-14.

<http://www.bioline.org.br/request?cn03001> Consulta: junio. 2005.

Sociedad Chilena de Enfermedades

Respiratorias en linea. Santiago de Chile. $<$ http://www.serchile.cl/enfermedades_tabaquism o.php> Consulta: junio. 2005.

Uribe, Alfonzo. "Prevalencia del tabaquismo en estudiantes universitarios" en línea. Revista Diagnóstico. 2001.

$<\mathrm{Http}$ ://www.fihu-diagnostico.org.pe/revista /numeros/2001/enefeb01/6-12.html> Consulta: junio. 2005. 